\title{
Rotationally resolved electronic spectroscopy of 4-aminobenzonitrile
}

\author{
Giel Berden ${ }^{\text {a }}$, Jack van Rooy ${ }^{a}$, W. Leo Meerts ${ }^{\text {a }}$, Klaas A. Zachariasse ${ }^{b}$ \\ "Department of Molecular and Laser Physics, University of Nijmegen. Toernooiveld, 6525 ED Nijmegen, The Netherlands \\ ${ }^{\mathrm{b}}$ Max-Planck-Institut für biophysikalische Chemie. D-37070 Göttingen, Germany
}

Received 24 February 1997; in final form 9 September 1997

\begin{abstract}
The rotationally resolved fluorescence excitation spectrum of the $0_{0}^{0}$ band in the $\mathrm{S}_{1} \leftarrow \mathrm{S}_{0}$ transition of 4-aminobenzonitrile (ABN) was recorded, at $299 \mathrm{~nm}$, by using laser induced fluorescence in a molecular beam apparatus. This spectrum exhibits pure $b$-type character, which indicates that the electronic transition moment vector is oriented along the short molecular axis. The rotational constants of the $S_{0}$ and $S_{1}$ states were determined. In addition, the rotationally resolved fluorescence excitation spectra of two vibronic bands in the $S_{1}$ state, at 807 and $816 \mathrm{~cm}^{-1}$, were recorded. The molecular structure of the ABN molecule is discussed by comparing the rotational constants and the inertial defects. (C) 1997 Elsevier Science B.V.
\end{abstract}

\section{Introduction}

Much attention has been devoted during the last years to the assignment of the vibrational modes in the laser induced fluorescence (LIF) excitation and emission spectra of jet-cooled 4-aminobenzonitrile (ABN) (Fig. 1) [1-4]. This assignment is of interest in connection with the dual fluorescence observed in polar and also in nonpolar solvents for 4-(dimethylamino)benzonitrile (DMABN) and related molecules, belonging to the same family as $\mathrm{ABN}$ [5]. It was found that the replacement of one or two methyl groups in DMABN by hydrogen atoms, giving respectively 4-(methylamino)benzonitrile (MABN) and $A B N$, results in the complete disappearance of the emission from the charge transfer (CT) state [6]. This phenomenon cannot be attributed to the increase in the oxidation potential of the amino groups $\mathrm{NH}\left(\mathrm{CH}_{3}\right)$ or $\mathrm{NH}_{2}$ relative to the dimethylamino substituent $[7,8]$. Such a behaviour is to be expected within the context of the twisted intramolecular charge transfer (TICT) model, which postulates that in the equilibrated emitting CT state of molecules such as DMABN the $\mathrm{N}\left(\mathrm{CH}_{3}\right)_{2}$ group is located in a plane perpendicular to the benzonitrile moiety [9]. The observation of single local excited state (LE) fluorescence in the case of $\mathrm{ABN}$ and MABN has initiated the introduction of an alternative model to describe the intramolecular charge transfer (ICT) and dual fluorescence of DMABN [10-14]. Attention has been drawn to the importance of the energy difference $\Delta E\left(S_{1}, S_{2}\right)$ between the two lowest excited singlet states $S_{1}$ and $S_{2}$ for the appearance of dual fluorescence [10-14]. It has also been suggested that the 

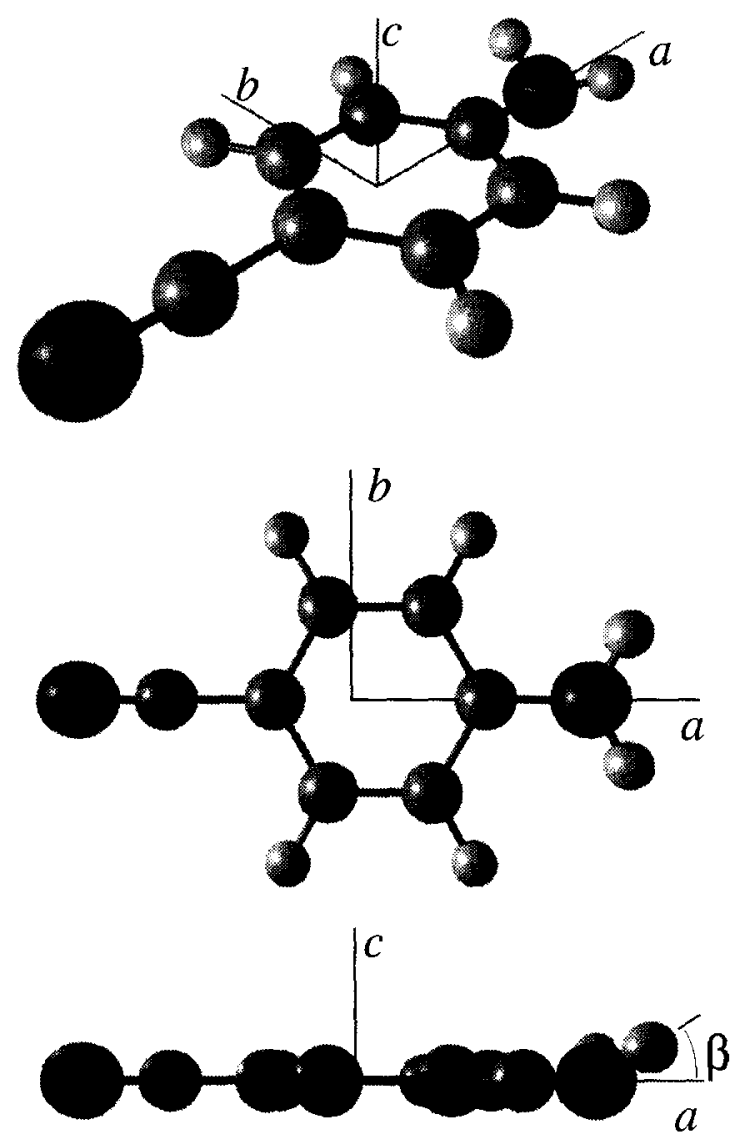

Fig. 1. Three different views of 4-aminobenzonitrile $(\mathrm{ABN})$ and its inertial axes. The angle $\beta$ designates the angle between the amino plane and the benzene plane.

change of the configuration of the amino group from pyramidal towards planar is an important ICT reaction coordinate [10-14].

Gibson et al. $[1,2]$ have shown that from a spectroscopical point of view ABN closely resembles aniline, rather than benzonitrile. It was further noticed that the amino inversion mode is very active in the excitation as well as the emission spectra. Recently, Yu et al. [4] used the semi-empirical quantum chemical parametric method PM3 to compute the geometry and the normal modes of $\mathrm{ABN}$ in its ground and first excited state. The normal modes calculated by this method were in good agreement with the experimental values. The calculated rotational constants have been used to simulate a rotational band contour of the origin band, which was compared with the experimental contour.
Accurate experimental values for the rotational constants are not available for ABN. Howells et al. [15] reported a rotational band contour study on jet cooled $\mathrm{ABN}$ at a resolution of $0.1 \mathrm{~cm}^{-1}$. They simulated the spectra by varying only the ground state constants, thereby keeping the differences between the constants in the excited and ground state fixed (i.e. $\Delta B=\Delta C=0$ and $\left(A^{\prime \prime}-A^{\prime}\right) / A^{\prime \prime}=0.03$ ) [15]. In this paper we report on the fully rotationally resolved UV fluorescence excitation spectra of the $0_{0}^{0}$ band and of two vibronic bands in the $S_{1} \leftarrow S_{0}$ transition of $\mathrm{ABN}$.

\section{Experimental}

Fluorescence excitation spectra of $\mathrm{ABN}$ were obtained by using a narrow bandwidth UV laser system and a molecular beam apparatus [16,17]. Crystalline ABN (Fluka, 97\%) was heated to approximately $120^{\circ} \mathrm{C}$ in a quartz oven. A molecular beam was formed by a continuous expansion of a mixture of ABN vapor and argon (500-600 Torr) through a nozzle with a diameter of $0.15 \mathrm{~mm}$. The nozzle was kept at a slightly higher temperature to prevent condensation of ABN in the orifice. The molecular beam was skimmed twice and then entered a differentially pumped LIF detection chamber at a distance of 30 $\mathrm{cm}$ from the nozzle outlet. At this position, the molecular beam was crossed perpendicularly with the weakly focused UV laser beam. The ABN molecules were resonantly excited from the $S_{0}$ to the $S_{1}$ state, and the total fluorescence of $S_{1}$ back to the electronic ground state was detected. Narrow band UV radiation was generated by intracavity frequency doubling a single frequency ring dye laser (a modified Spectra Physics 380D) operating on rhodamine 6G. By using a $\mathrm{LiIO}_{3}$ crystal ( $1.5 \mathrm{~mm}$ thick), $5 \mathrm{~mW}$ of tunable radiation was obtained with a wavelength around $299 \mathrm{~nm}$ and an effective bandwidth of 3 $\mathrm{MHz}$. Due to self-absorption of UV radiation at wavelengths below $300 \mathrm{~nm}, \mathrm{LiIO}_{3}$ cannot be used for generating UV light below $295 \mathrm{~nm}$. To cover the wavelength range around $291.5 \mathrm{~nm}$, a $2 \mathrm{~mm}$ thick BBO crystal was used $(200-500 \mu \mathrm{W})$. The excitation spectra of $A B N$ were recorded together with the transmission peaks of a pressure and temperature stabilized interferometer with a free spectral range of 


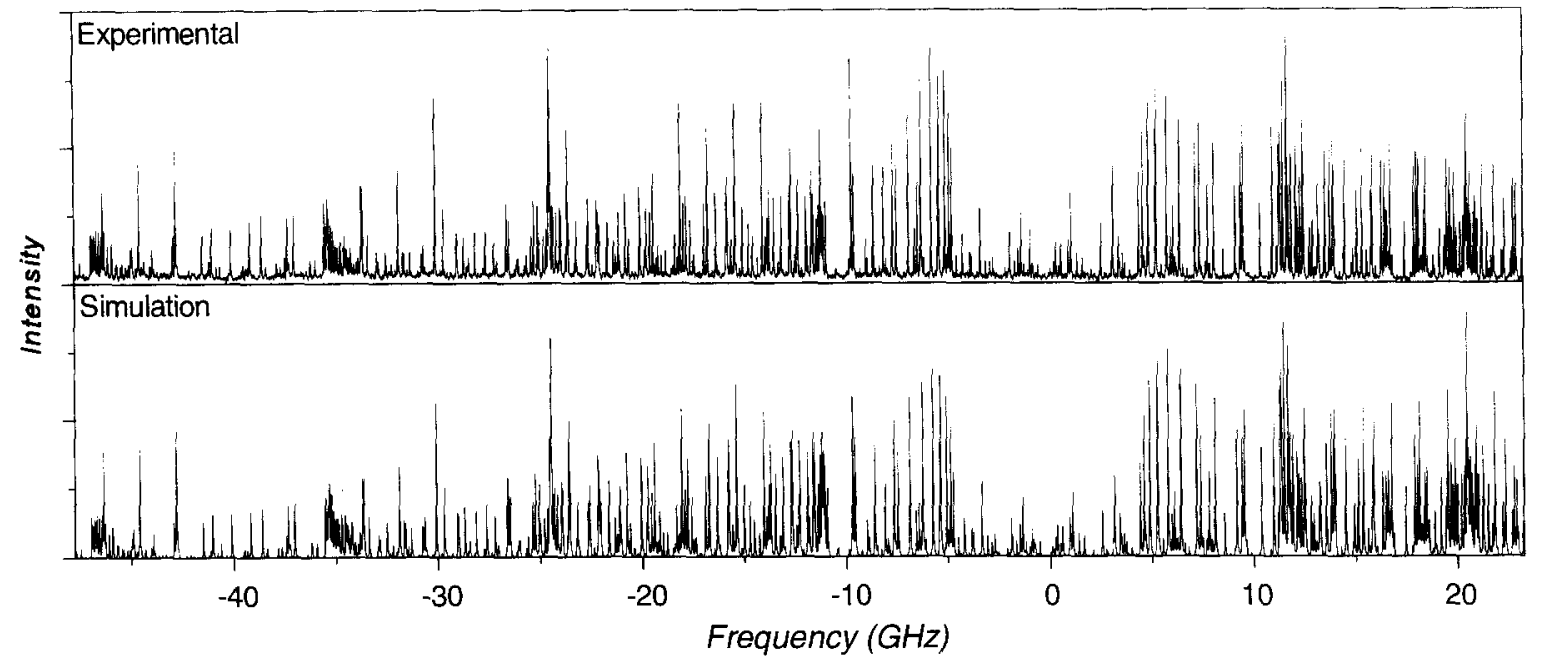

Fig. 2. Part of the high resolution LIF spectrum of the $0_{0}^{0}$ band of the $S_{1} \leftarrow S_{0}$ transition of 4-aminobenzonitrile (ABN). Both the experimental and the simulated spectra are shown. The absolute frequency of the origin $(0.0$ on the scale of the figure $)$ is at $33481.440 \pm 0.003 \mathrm{~cm}^{-1}$.

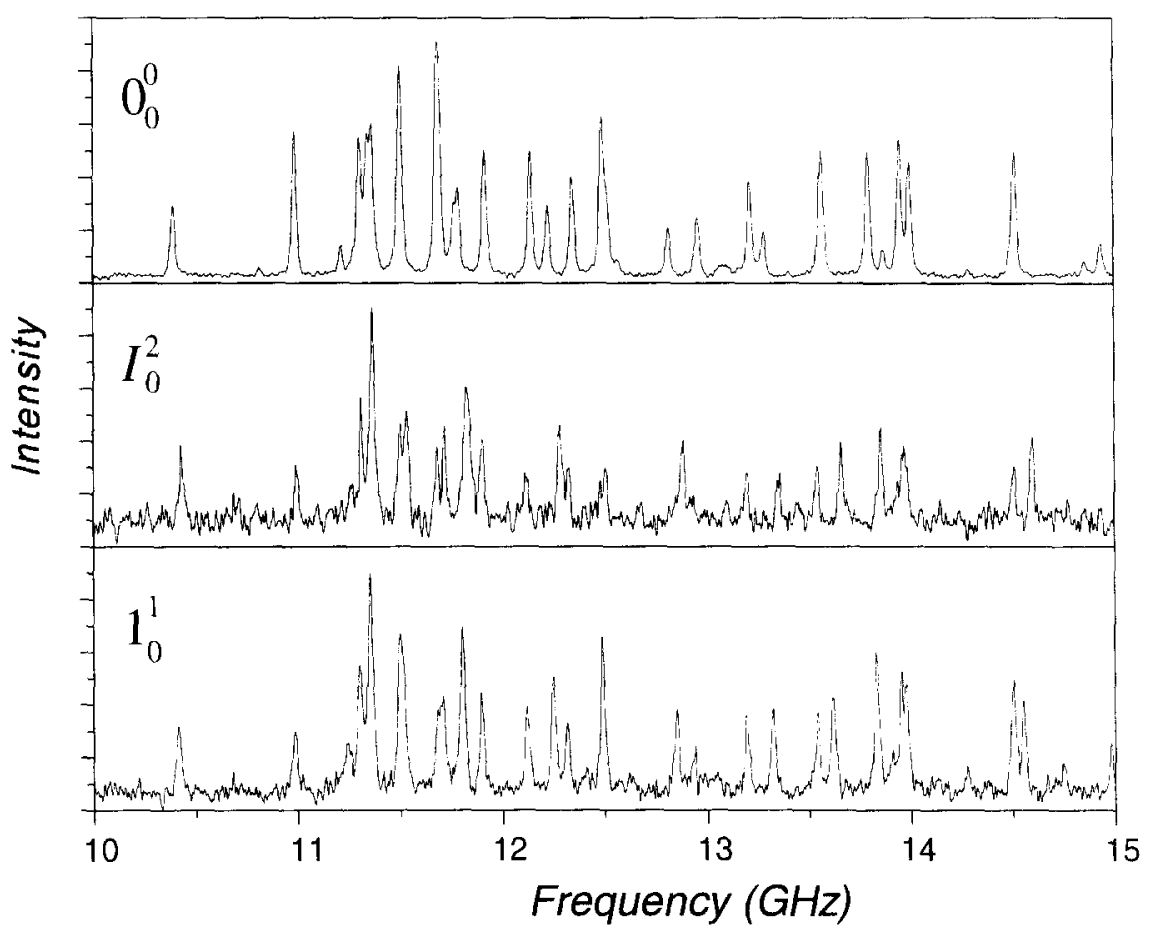

Fig. 3. Details of the spectra of the $0_{0}^{0}$, the $I_{0}^{2}$, and the $1_{0}^{1}$ band of $\mathrm{ABN}$. The origin of each spectrum is set at $0 \mathrm{GHz}$ (the absolute frequencies are listed in Table 1). Differences between the spectra are due to the small differences between the rotational constants of the bands in the $S_{1}$ state. 
$75 \mathrm{MHz}$. For absolute frequency calibration, the iodine absorption spectrum $[18,19]$ was recorded.

\section{Results}

In Figs. 2 and 3 (upper panel) the high resolution LIF excitation spectrum of the $0_{0}^{0}$ band of the $S_{1} \leftarrow$ $S_{0}$ transition in ABN is shown. The absolute frequency of the band origin ( 0.0 on the scale of the figures) is at $33481.440 \pm 0.003 \mathrm{~cm}^{-1}$. The spectrum consists of about 800 well resolved lines with a linewidth of $26 \mathrm{MHz}$ and the recording time was less than 10 minutes. In this way the drift of the interferometer during the scan was minimized.

The spectrum can immediately be identified as a $b$-type band. This indicates that the electronic transition moment vector in $\mathrm{ABN}$ is oriented parallel to the inertial $b$-axis (polarized along the short axis), as has also been observed for aniline [20-22] and benzonitrile [23].

After the identification of the band type, the following procedure was used to analyze the data. A spectrum was simulated by using an asymmetric rotor Hamiltonian and employing rotational constants obtained from a crude geometrical structure. The spectrum obtained in this way was then compared with the experimental spectrum. An initial assignment was made in the center part of the spectrum, which has the lowest spectral density. The assigned lines were used as input for the fitting program. With the improved rotational constants the spectrum was simulated again and compared with the experimental spectrum. This procedure was repeated several times. Finally, 350 lines were included in the fit, all parameters being varied simultaneously. All lines could be fitted within the experimental error.

\footnotetext{
${ }^{1}$ The inertial defect $\Delta I$ in a specific state is defined as $\Delta I=I_{c}-I_{b}-I_{a}$ in which $I_{g}$ are the principal moments of inertia $(g=a, b, c)$. The inertial defect is zero when the molecule is planar. However due to zero-point vibrations the actual value of the inertial defect will slightly differ from zero; out-of-plane (in-plane) motions give a negative (positive) contribution to the inertial defect. The rotational constants are inverse proportional to the principal moments of inertia (i.e., $A \propto 1 / I_{a}$ ). More detailed information can be found in Ref. [24]
}

Table 1

Molecular constants of 4-aminobenzonitrile (ABN). The rotational constants (in $\mathrm{MHz})$ and the inertial defect $\left(\Delta I=I_{c}-I_{b}-I_{a}\right.$, in amu $\AA^{2}$ ) in the $S_{0}$ state, the differences of the rotational constants between the $S_{1}$ and $S_{0}$ states $\left(\Delta A=A^{\prime}-A^{\prime \prime}\right.$, etc.), and the inertial defect in the $S_{1}$ state. The constants are listed for three bands, which have their origin at the absolute frequency $\nu_{0}$ (in $\mathrm{cm}^{-1}$ )

\begin{tabular}{|c|c|c|c|c|c|}
\hline & \multirow[t]{2}{*}{$\mathrm{S}_{0}$} & \multicolumn{4}{|c|}{$S_{1}$} \\
\hline & & & 0 & $I_{0}^{2}$ & $1_{0}^{1}$ \\
\hline$A^{\prime \prime}$ & $5579.3(5)$ & $\Delta A$ & $-316.61(6)$ & $-323.97(5)$ & $-323.99(10)$ \\
\hline$B^{\prime \prime}$ & $990.26(9)$ & $\Delta B$ & $10.849(3)$ & $11.392(3)$ & $11.082(7)$ \\
\hline$C^{\prime \prime}$ & $841.39(8)$ & $\Delta C$ & $0.095(1)$ & $0.989(2)$ & $0.557(5)$ \\
\hline$\Delta I^{\prime \prime}$ & $-0.293(2)$ & $\Delta I^{\prime}$ & $-0.281(2)$ & $-0.878(3)$ & $-0.608(9)$ \\
\hline$\nu_{0}$ & & & $33481.440(3)$ & $34288.676(3)$ & $34297.760(3)$ \\
\hline$\Delta \nu_{0}$ & & & 0.0 & 807.236 & 816.320 \\
\hline
\end{tabular}

The absolute precision of the UV data is limited by thermal drift of the frequency markers. The magnitude of this error can be determined by scanning the spectrum several times on different days. From these fits, we obtain the origin of the transition and the rotational constants $A, B$, and $C$ in the ground state as well as in the excited state. The constants are listed in Table 1 together with the asymmetry parameters and the inertial defects ${ }^{1}$ [24].

The shape of the spectrum depends on the rotational temperature and on the linewidth of the individual rotational lines. The best simulation of the experimental spectrum was obtained with a rotational temperature of $3 \mathrm{~K}$ and a linewidth of 26 $\mathrm{MHz}$, and is shown in the lower panel of Fig. 2. The linewidth of our spectrometer is known to be 14 $\mathrm{MHz}$, caused by residual Doppler broadening, transit time effects, fluorescence collection optics and laser linewidth. This comparison shows that our experimental linewidth contains a noticeable contribution from the finite excited state lifetime of ABN. Deconvolution of the lineshapes of several isolated rotational transitions yields this life time broadening. The lifetime $\tau$ of the origin can then be determined to be $9 \pm 3 \mathrm{~ns}$, which is slightly less than the reported value of $13.0 \mathrm{~ns}$ [3].

Besides the origin band, we have measured the rotationally resolved excitation spectra of two vibronic bands at 807 and $816 \mathrm{~cm}^{-1}$ above the electronic origin (see Fig. 3). The first band has been assigned by Gibson et al. [2] to the $\Delta v=2$ transition 
in the amino group inversion mode, $I_{0}^{2}$. The signal to noise ratio of the high resolution spectrum of this band is worse than that of the spectrum of the origin band, for which two reasons can be given. First, the Franck-Condon factor of the $I_{0}^{2}$ band is small. In the low resolution spectrum, this band is a factor two lower in intensity than the origin band [3]. Secondly, the power of the excitation laser is a factor $10-20$ lower as a result of the use of a $\mathrm{BBO}$ crystal for generating UV, rather than the more efficient $\mathrm{LiIO}_{3}$ which could be used to measure the origin band. The other vibronic band, at $816 \mathrm{~cm}^{-1}$, has been assigned to the $\Delta v=1$ transition in the ring breathing mode, $1_{0}^{1}[2,4]$. Both vibronic bands are of $b$-type. All lines in both spectra, could be assigned and fitted to an asymmetric rotor Hamiltonian. The rotational constants are presented in Table 1. The linewidths of the $I_{0}^{2}$ and the $1_{0}^{1}$ bands are $24 \mathrm{MHz}$, which results in a value for the life time of $9 \pm 4 \mathrm{~ns}$, after deconvolution. This value is in agreement with the reported life time for the $I_{0}^{2}$ band (10.9 ns) and for the $1_{0}^{1}$ band (11.1 ns) [3].

\section{Discussion}

Although it is not possible to determine the complete structure of $\mathrm{ABN}$ from our data, we can derive a qualitative picture of the structure in the $S_{0}$ state and of the structural changes upon excitation to the $\mathrm{S}_{1}$ state. The inertial defect $\Delta I$ in the $\mathrm{S}_{0}$ state of $\mathrm{ABN}$ is $-0.293(2)$ amu $\AA^{2}$. This value can be compared to those found for benzonitrile, $+0.093(30)$ amu $\AA^{2}$ [25], and aniline, $-0.408(1)$ amu $\AA^{2}$ [26], for which the complete substitution structures have been determined ( [25] and [27], respectively). Benzonitrile is planar in the ground state [25]. In aniline, the hydrogen atoms of the $\mathrm{NH}_{2}$ group are not coplanar with the rest of the molecule. The inversion angle $\beta$ of this group with respect to the plane of the phenyl ring has been determined to be $37^{\circ}-44^{\circ}[26-28]$. The inertial defect observed for $\mathrm{ABN}$ is smaller than that of aniline, which would suggest that the angle $\beta$ of $\mathrm{ABN}$ is slightly smaller (by ca. $6^{\circ}$ ).

The structure of $\mathrm{ABN}$ in the crystalline phase (at $153 \mathrm{~K})$ has been determined by Heine et al. [29]. They concluded that the inversion angle $\beta$ amounts
Table 2

Rotational constants of 4-aminobenzonitrile. The experimental values in the gas-phase are from the present work. The crystal data are taken from Ref. [29]. The theoretical values, obtained from semi-empirical calculations, have been reported by $\mathrm{Yu}$ et al. [4]. The rotational constants are in $\mathrm{MHz}$, the inertial defects $\Delta /$ in amu $\AA^{2}$

\begin{tabular}{cccc}
\hline & Gas-phase & Crystal-phase & PM3 \\
\cline { 1 - 2 }$A^{\prime \prime}$ & $5579.3(5)$ & 5756.4 & 5527.5 \\
$B^{\prime \prime}$ & $990.26(9)$ & 1006.8 & 994.7 \\
$C^{\prime \prime}$ & $841.39(8)$ & 857.3 & 843.8 \\
$\Delta A$ & $-316.61(6)$ & & -155.3 \\
$\Delta B$ & $10.849(3)$ & & 6.4 \\
$\Delta C$ & $0.095(1)$ & & 0.3 \\
$\Delta I^{\prime \prime}$ & $-0.293(2)$ & -0.28 & -0.59 \\
$\Delta I^{\prime}$ & $-0.281(2)$ & & -0.14 \\
\hline
\end{tabular}

to $34(3)^{\circ}$. The nitrogen atom of the amino group is located out of the benzene plane by $0.059(3) \AA$ (in a direction opposite to the amino hydrogen atoms). The cyano group likewise is not coplanar with the phenyl ring, with an angular orientation on the same side as the amino hydrogen atoms ( $\mathrm{C}$ atom $0.020(3)$ $\AA$, N atom $0.047(3) \AA$ ). From the positions of all atoms, the rotational constants and the inertial defect were calculated (Table 2). Since bond lengths of molecules in the crystalline phase are often slightly smaller than those in the gas phase ${ }^{2}$, the rotational constants of crystalline $\mathrm{ABN}$ are expected to be larger than those in the gas-phase. It is important to note, however, that the inertial defects are found to be equal for both phases. This indicates that the out-of-plane atomic positions of $\mathrm{ABN}$ in the gasphase can be reasonable well described by the positions in the crystal. The out-of-plane positions of the nitrogen atoms give only a minor contribution to the inertial defect. We therefore conclude that the inversion angle $\beta$ of $\mathrm{ABN}$ is approximately equal to $34^{\circ}$. Note that the inversion angle of aniline in the crystalline phase is determined to be $38(3)^{\circ}$ [30] similar to the reported gas phase values $\left(37^{\circ}-44^{\circ}[26-28]\right)$ as well.

Upon electronic excitation to the origin of the $S_{1}$ state, the $A$ constant of $\mathrm{ABN}$ decreases with $5.7 \%$.

\footnotetext{
${ }^{2}$ Compare, for example, the bondlengths obtained from microwave (gas phase) and X-ray (crystalline phase) studies of aniline $[27,30]$.
} 
the $B$ constant increases with $1.1 \%$, while the $C$ constant remains practically unchanged. A comparison of the changes in the rotational constants of $\mathrm{ABN}$ with those of aniline $(\Delta A=-5.9 \%, \Delta B=$ $+1.5 \%, \Delta C=-1.0 \%[21,22])$, and benzonitrile $(\Delta A=-3.2 \%, \Delta B=-2.3 \%, \Delta C=-2.5 \%$ [23]), shows that $A B N$ closely resembles aniline, rather than benzonitrile. A similar conclusion was drawn by Gibson et al. on the basis of an analysis of the vibrationally resolved electronic excitation and emission spectra [1,2]. However, the inertial defect of aniline decreases upon excitation (from $-0.408 \mathrm{amu}$ $\AA^{2}$ in the $S_{0}$ state to -0.241 amu $\AA^{2}$ in the $S_{1}$ state [22,21]), while the inertial defect of $A B N$ remains constant $\left(-0.293\right.$ amu $\AA^{2}$ in the $S_{0}$ and -0.281 amu $\AA^{2}$ in the $S_{1}$ state).

Qualitatively, the changes in the rotational constants can be explained by an expansion of the benzene ring of $\mathrm{ABN}$ (with the two $\mathrm{C}-\mathrm{C}$ bonds parallel to the $a$-axis increasing less than the other four $\mathrm{C}-\mathrm{C}$ bonds), a contraction of the $\mathrm{C}-\mathrm{N}$ (amino) bond, and a slight reduction of the $\mathrm{C}-\mathrm{N}$ (cyano) bond length. Since the inertial defect in the $S_{1}$ state is almost identical to that of the $S_{0}$ state, as mentioned earlier, it is concluded that the inversion angle $\beta$ remains unchanged upon excitation. In aniline, however, the inversion angle decreases upon electronic excitation resulting in a quasi planar structure in the $S_{1}$ state [22]. Therefore, assuming that the aniline part of $A B N$ is quasi planar in the $S_{1}$ state as well, the inertial defect would be due to an out-of-plane motion of the cyano group or to an increased contribution of the zero-point vibrations.

Exciting $\mathrm{ABN}$ to the $I^{2}$ or $1^{1}$ state, leads to a slight increase of the absolute values of the differences in rotational constants (compared to those obtained for the origin). Similar changes have been observed for the $I_{0}^{2}$ and $1_{0}^{1}$ bands of aniline $[22,31]$. The value of the inertial defect of the $I^{2}$ state for ABN $\left(-0.878\right.$ amu $\left.\AA^{2}\right)$ is remarkable close to the value for aniline $\left(-0.868\right.$ amu $\left.\AA^{2}\right)$, and is more negative than the inertial defect of the $0^{0}$ state as expected for an out-of-plane mode. The situation is different for the ring breathing mode. The inertial defect of the $1^{1}$ state of aniline is -0.20 amu $\AA^{2}$ (similar to that of the $0^{0}$ state of aniline), while the inertial defect of the $1^{1}$ state of $\mathrm{ABN}$ is $-0.608 \mathrm{amu}$ $\AA^{2}$. This indicates that there is an additional out-of- plane motion (probably the cyano group) associated with the ring breathing vibration since the inertial defect is more negative than that of the $0^{0}$ state of ABN.

The rotational constants listed in Table 1 can be taken to test the results of theoretical calculations. Yu et al. [4] used the semi-empirical quantum chemical calculation PM3 to compute the geometry and the normal modes of $A B N$ in the $S_{0}$ and $S_{1}$ states. The calculated normal modes were in good agreement with the experimental values [4]. The calculated rotational constants can now be compared with the experimental constants of the present study (Table 2). It is seen that the calculated ground state constants are in good agreement with the experimental values. The agreement is worse for the excited state. Furthermore, the calculations predict a decrease in the inertial defect which is not observed. It is interesting to note that a similar decrease (although of smaller magnitude) has been observed for aniline $[21,22]$.

The results of present study can be useful for the analysis and interpretation of derivatives of $A B N$ with a more complex structure. An important derivative is DMABN. This molecule and related compounds are under investigation because of their dual fluorescence observed in polar as well as in non polar solvents [6-14]. Rotationally resolved LIF spectra of such derivatives would give access to information about the geometrical structure in both the ground and electronically excited state. Unfortunately, the measured high resolution excitation spectra of DMABN are heavily perturbed by torsional motions of the methyl groups $[32,33]$ and an analysis as carried out here for $A B N$ will be more complicated.

\section{Acknowledgements}

This work was made possible by financial support from the Dutch Foundation for Fundamental Research on Matter (FOM).

\section{References}

[1] E.M. Gibson, A.C. Jones, A.G. Taylor, W.G. Bouwman, D. Phillips, J. Phys. Chem. 92 (1988) 5449. 
[2] E.M. Gibson, A.C. Jones, D. Phillips, Chem. Phys. Lett. 146 (1988) 270.

[3] H. Yu, E. Joslin, B. Crystall, T, Smith, W. Sinclair, D. Phillips, J. Phys. Chem. 97 (1993) 8146

[4] H. Yu, E. Joslin, S.M. Zain, H. Rzepa, D. Phillips, Chem. Phys. 178 (1993) 483.

[5] W. Rettig, Angew. Chem. Int. Ed. Engl. 25 (1986) 971.

[6] U. Leinhos, W. Kühnle, K.A. Zachariasse, J. Phys. Chem. 95 (1991) 2013

[7] K.A. Zachariasse, Th. von der Haar, A. Hebecker, U. Leinhos, W. Kühnle, Pure \& Appl. Chem. 65 (1993) 1745.

[8] W. Schuddeboom, S.A. Jonker, J.M. Warman, U. Leinhos, W. Kühnle, K.A. Zachariasse, J. Phys. Chem. 96 (1992) 10809.

[9] E. Lippert, W. Rettig, V. Bonac̃ić-Koutecký, F. Heisel, J.A. Miehé, Adv. Chem. Phys. 68 (1987) I.

[10] Th. von der Haar, A. Hebecker, Yu. Il'ichev, Y.-B. Jiang, W. Kühnle, K.A. Zachariasse, Recl. Trav. Chim. Pays-Bas 114 (1995) 430

[11] K.A. Zachariasse, Th. von der Haar, U. Leinhos, W. Kühnle, J. Inf. Recording 21 (1994) 501.

[12] Th. von der Haar, A. Hebecker, Yu. Il'ichev, W. Kühnle, K.A. Zachariasse, Fast elementary processes in chemical and biological systems, Lille. France, 1995, AIP Conference Proceedings 364 (1996) 295.

[13] K.A. Zachariasse. Th. von der Haar, U. Leinhos, W. Kühnle, J. Inf. Recording 22 (1996) 553.

[14] K.A. Zachariasse, M. Grobys, Th. von der Haar, A. Hebecker, Yu.V. Il'ichev, Y.-B. Jiang, O. Morawski, W. Kühnle, J. Photochem. Photobiol. A: Chem. 105 (1997) 373.
[15] B.D. Howells, J. McCombie, T.F. Palmer, J.P. Simons, A. Walters, J. Chem. Soc. Faraday Trans. 88 (1992) 2595.

[16] W.A. Majewski, W.L. Meerts, J. Mol. Spectrosc. 104 (1984) 271.

[17] G. Berden, Ph.D. Thesis, University of Nijmegen, 1995.

[18] S. Gerstenkorn, P. Luc, Atlas du spectroscopie d'absorption de la molecule d'iode, CNRS, Paris, 1978.

[19] S. Gerstenkorn. P. Luc, Rev. Phys. Appl. 14 (1979) 791.

[20] J. Christoffersen, J.M. Hollas, G.H. Kirby. Mol. Phys. 16 (1969) 441.

[21] E.R.Th. Kerstel, M. Becucci, G. Pietraperzia, E. Castellucci, Chem. Phys, 199 (1995) 263.

[22] W.E. Sinclair, D.W. Pratt, J. Chem. Phys. 105 (1996) 7942.

[23] J.C.D. Brand, P.D. Knight, J. Mol. Spectrose. 36 ( 1970) 328.

[24] W. Gordy, R.L. Cook, Microwave Molecular Spectra, 3rd ed., Wiley, New York, 1984.

[25] B. Bak, D. Christensen, W.B. Dixon, L. Hansen-Nygaard, J. Rastrup-Andersen, J. Chem. Phys. 37 (1962) 2027.

[26] B. Kleibömer, D.H. Sutter, Z. Naturforsch. 43a (1988) 561.

[27] D.G. Lister, J.K. Tyler, J.H. Høg. N.W. Larsen, J. Mol. Struct. 23 (1974) 253.

[28] M. Quack, M. Stockburger. J. Mol. Spectrosc. 43 (1972) 87.

[29] A. Heine. R. Herbst-Irmer, D. Stalke. W. Hühnle, K.A. Zachariasse, Acta Cryst. B 50 (1994) 363.

[30] M. Fukuyo, K. Hirotsu, T. Higuchi, Acta Cryst. B 38 (1982) 640.

[31] E.R.Th. Kerstel, M. Becucci, G. Pietraperzia, D. Consalvo. E. Castellucci, J. Mol. Spectrosc. 177 (1996) 74.

[32] G. Berden. unpublished results.

[33] D.W. Pratt, Pittsburgh, private communication. 